\title{
What are the low- $Q$ and large- $x$ boundaries of collinear QCD factorization theorems?
}

\author{
E. Moffat* \\ Old Dominion University \\ E-mail: emoff003@odu.edu \\ T. C. Rogers \\ Old Dominion University \& Jefferson Lab \\ E-mail: tedconantrogers@gmail.com \\ W. Melnitchouk \\ Jefferson Lab \\ E-mail: wmelnitc@jlab.org \\ N. Sato \\ Jefferson Lab \\ E-mail: nsatodjlab.org
}

We demonstrate testing QCD factorization theorems using a simple quantum field theory. Specifically we test standard collinear factorization in order to better explore deeply inelastic scattering (DIS) at lower $Q$ and larger Bjorken $x$.

QCD Evolution 2017

22-26 May, 2017

Jefferson Lab Newport News, VA - USA

${ }^{*}$ Speaker. 


\section{Introduction}

It is of interest to explore the physics of DIS at lower values of momentum transfer $(Q)$ and larger values of momentum fraction $\left(x_{\mathrm{bj}}\right)$. At lower ranges of $Q, \alpha_{s}$ may still be small, but effects from beyond the usual kinematical collinear approximations become important. These include such effects as target mass, higher twist corrections, parton transverse momentum, and parton virtuality. It is difficult to ascertain the relative importance of these effects in QCD due to confinement and asymptotic freedom. However, by applying QCD factorization to a simple quantum field theory whose calculations require no approximations, we can test the relative size of the contributions of these effects. What follows is a summary of how that can be done. For the complete analysis, see [1].

\section{Simple Field Theory}

We use a simple theory describing the interaction between a spin-1/2 "nucleon" with mass $M$ represented by the field $\Psi_{N}$, a spin-1/2 "quark" field $\psi_{q}$ with mass $m_{q}$, and a scalar "diquark" state $\phi$ with mass $m_{s}$ that does not couple to the photon but remains a spectator to the hard scattering from the quark. The interaction Lagrangian density for this theory is given by a Yukawa-like interaction,

$$
\mathscr{L}_{\text {int }}=-\lambda \bar{\Psi}_{N} \psi_{q} \phi+\text { H.c. }
$$

where the coupling $\lambda$ gives the strength of the nucleon-quark-diquark interaction.

In this model, we calculate a $k_{\mathrm{T}}$-unintegrated structure function $\mathscr{F}_{1}$ defined as

$$
F_{1}\left(x_{\mathrm{bj}}, Q^{2}\right)=\int \frac{\mathrm{d}^{2} k_{\mathrm{T}}}{(2 \pi)^{2}} \mathscr{F}_{1}\left(x_{\mathrm{bj}}, Q^{2}, k_{\mathrm{T}}^{2}\right),
$$

where $x_{\text {bj }}$ is Bjorken- $x$. We first calculate $\mathscr{F}_{1}$ without making any approximations. The contributing diagrams are shown in Fig. 1. We then calculate the same function after applying the appropriate
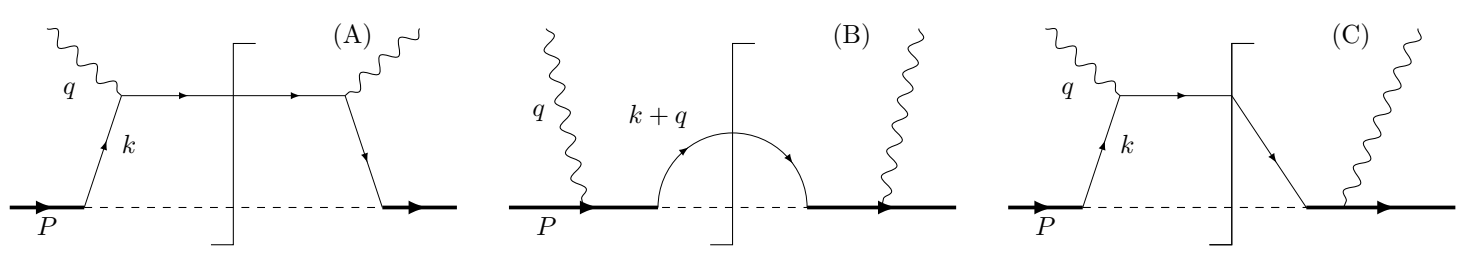

Figure 1: Contributions to the hadronic tensor from diagrams allowed by the interaction Lagrangian (2.1) to $O\left(\alpha \lambda^{2}\right)$ in the couplings. Graph (A) is a manifestation of the familiar handbag diagram and represents the topology of the leading region. Graphs (B) and (C) are suppressed by powers of $1 / Q$ when $k_{\mathrm{T}}$ is small, but are needed for gauge invariance. The Hermitian conjugate for $(C)$ is not shown. The momenta on the various legs are as indicated.[1]

approximations of standard collinear factorization. For details of the calculations, see [1]

\section{Results}

Fig. 2 is a set of plots showing the exact and approximated structure functions vs. $k_{\mathrm{T}}$ evaluated at $Q$ of 2 and $20 \mathrm{GeV}$ with various values of the quark and spectator masses. See [1] for explanation 
of the specific choices of masses. From these results, it is clear that at lower $Q$, the agreement between the exact and approximate structure functions is not only dependent on the transverse momentum but also the mass and virtuality of the struck parton.
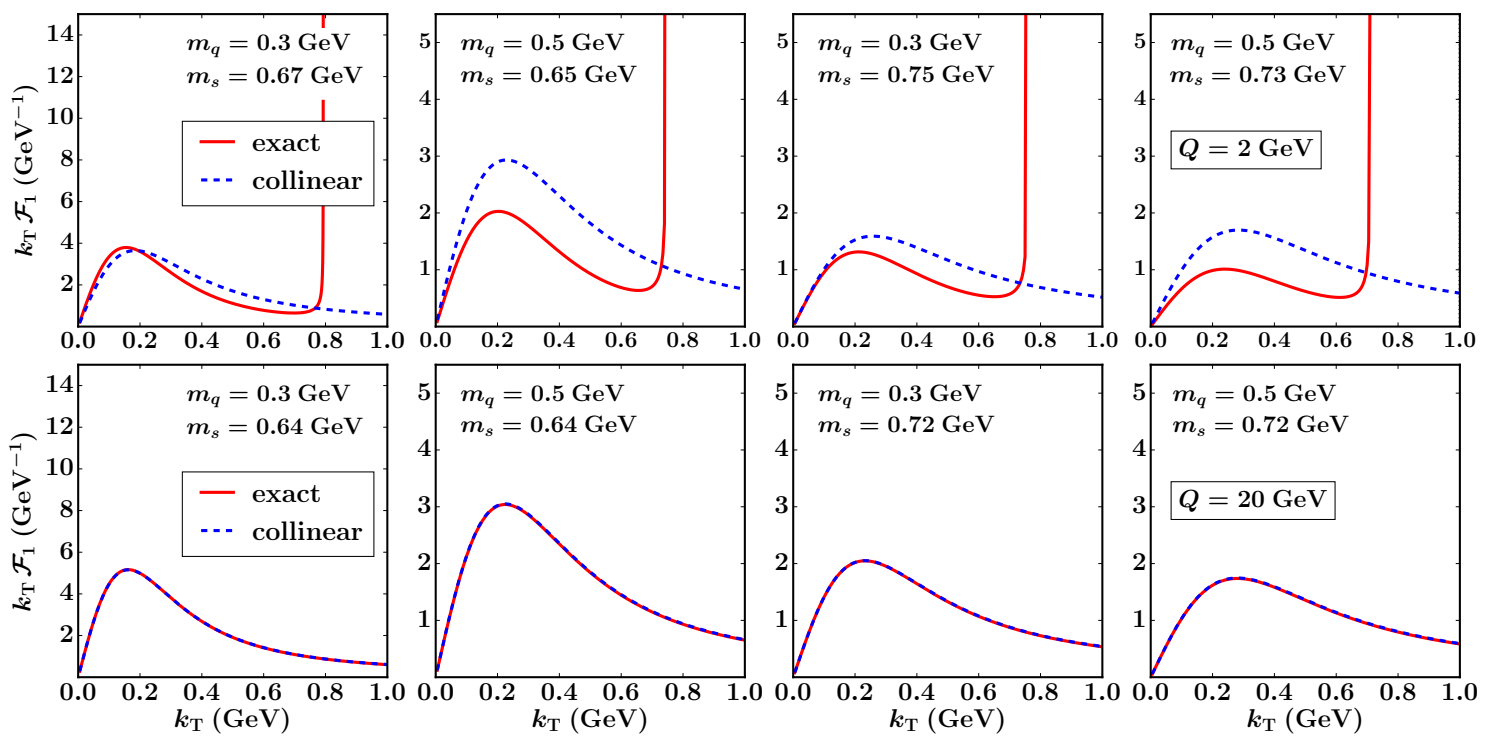

Figure 2: The unintegrated structure function $k_{\mathrm{T}} \mathscr{F}_{1}$ for $x_{\mathrm{bj}}=0.6$ and $Q=2 \mathrm{GeV}$ (top row) and $Q=20 \mathrm{GeV}$ (bottom row), for different values of $m_{q}$ and $m_{s}$ calculated using both the exact expressions (solid red curves) and the canonical collinear factorization approximation (dashed blue curves). The choices of $m_{s}$ are to fix $k^{2}$ at the values discussed in [1]. At the higher $Q$ value the collinear calculation is almost indistinguishable from the exact, while at the lower $Q$ value the exact calculation diverges as it approaches the kinematical upper limit of $k_{\mathrm{T}} \cdot[1]$

\section{Conclusion}

Analysis using the simple QFT demonstrates that the most accurate QCD factorization theorem for low- $Q$ and large- $x_{\mathrm{bj}}$ would need to account for corrections due to parton mass, parton transverse momentum, and parton virtuality as well as the target mass.

\section{References}

[1] E. Moffat, W. Melnitchouk, T. C. Rogers and N. Sato, What are the low-Q and large- $x$ boundaries of collinear QCD factorization theorems?, Phys. Rev. D95 (2017) 096008, [1702 . 03955]. 